NOTAS Y EXPERIENCIAS / NOTES AND EXPERIENCES

\title{
Competencias profesionales de los postgraduados en el Máster de Gestión de la Documentación, Bibliotecas y Archivos desde la perspectiva de empleadores, estudiantes y profesores
}

\author{
Alicia Arias-Coello*, Clara Simón-Blas**, José Simón-Martín*** \\ *Facultad de Ciencias de la Documentación. Universidad Complutense de Madrid. España. \\ **Departamento de Estadística e Investigación Operativa. Universidad Rey Juan Carlos, Madrid. España \\ ***Instituto Universitario de Evaluación Sanitaria. Universidad Complutense de Madrid. España \\ Correo-e: aarias@pdi.ucm.es
}

Recibido: 12-09-2013; 2a versión: 9-12-2013; Aceptado: 7-01-2014.

Cómo citar este artículo/Citation: Arias-Coello, A.; Simón-Blas, C.; Simón-Martín, J. (2014). Competencias profesionales de los postgraduados en el Máster de Gestión de la Documentación, Bibliotecas y Archivos desde la perspectiva de empleadores, estudiantes y profesores. Revista Española de Documentación Científica, 37(3):e059. doi: http://dx.doi.org/10.3989/ redc.2014.3.1121

Resumen: El presente estudio ha sido realizado para identificar en qué medida el Programa de Máster en Gestión de la Documentación, Bibliotecas y Archivos, ofertado por la Facultad de Ciencias de la Documentación de la UCM, incluye un conjunto de competencias consideradas como importantes por los empleadores, los profesores y los estudiantes del máster. Con este fin, se elaboró un cuestionario que fue enviado a empleadores del sector de la documentación y a profesores y estudiantes del máster para que valorasen la importancia de 29 competencias. A los profesores y estudiantes se les solicitó, además, su opinión acerca del grado en que dicha competencia estaba incluida en el máster. Como resultado más destacable podemos señalar: la existencia de una diferencia significativa en las valoraciones que hacen los estudiantes sobre la importancia de cada competencia y la satisfacción con su aprendizaje. El modelo de evaluación presentado permite, además, identificar las deficiencias en el currículo del máster y establecer las recomendaciones para su mejora.

Palabras clave: Máster en Gestión de la Documentación; bibliotecas y archivos; competencias de los profesionales de la información y la documentación; competencias máster; programas máster; encuesta a empleadores y estudiantes; satisfacción de estudiantes.

Professional competencies of graduates of the Master's in library and information sciences from the perspective of employers, professors and students

Abstract: This study was designed to identify the extent to which the Master's Program in Library and Information Science, offered by the school of Documentation Sciences at the Universidad Complutense de Madrid (Spain) covers the competencies considered important by employers and Master's students and professors. A questionnaire was prepared and sent to employers in the information science sector and to professors and students from the Master's Program, asking them to evaluate 29 competencies. Professors and students were also asked to indicate to what extent these skills are included in Master. The most notable result is the existence of a significant gap in the ratings made by students about the importance of each competency and their satisfaction with what was learned. The evaluation framework presented can be used to identify deficiencies in the Master's curricula and to make recommendations for improvement.

Keywords: Master in library and information sciences; information professional's competencies; master's competencies; master's program; employer's and student's survey; student's satisfaction.

Copyright: (c) 2014 CSIC. Este es un artículo de acceso abierto distribuido bajo los términos de la licencia Creative Commons Attribution-Non Commercial (by-nc) Spain 3.0. 


\section{INTRODUCCIÓN}

Con la implantación en España del Espacio Europeo de Educación Superior (EEES) en 2006, los estudios de Biblioteconomía y Documentación sufrieron un cambio de orientación para la adaptación al mismo. Esta adaptación implicó el diseño de los nuevos planes de estudio de grado y máster, y la exigencia de especificar las competencias que deben ser adquiridas por los estudiantes durante su proceso de aprendizaje.

Para un correcto diseño o rediseño de los planes de estudio y de los programas formativos en información, biblioteconomía y documentación, es necesario contar con el punto de vista de todas las partes implicadas: estudiantes, profesores, empleadores y profesionales.

Existen diversas fuentes documentales en el ámbito profesional, que son importantes en lo que se refiere a la definición de las competencias que deben poseer los egresados de las titulaciones pertenecientes a este área de conocimiento; así, en Europa, disponemos de un documento elaborado por un conjunto de profesionales perteneciente a diversas asociaciones, las cuales participaron en el Proyecto CERTIDOC. Este documento versa sobre el Desarrollo de las Euro-competencias en Información y Documentación (SEDIC, 2005) y, en su concepción, tuvo como objetivo el promover la cooperación entre todos los socios del proyecto para conseguir un mejor conocimiento de las competencias en el campo profesional de la información y la documentación, así como la adaptación de éstas al desarrollo tecnológico, a la competitividad de las empresas y a las necesidades del mercado de trabajo (Tejada y Meyriat, 2003).

Además, es importante destacar que otras organizaciones internacionales con gran influencia en este sector profesional, disponen de directrices o estándares sobre esta materia, como por ejemplo, la Federación Internacional de Asociaciones y Organizaciones de Bibliotecarios (IFLA, 2003), la Special Libraries Association (SLA), o los estándares utilizados por la American Library Association para la Acreditación de Programas de Máster en LIS (ALA, 2008).

Diversos investigadores han analizado las competencias que deberían poseer los profesionales de la información y la documentación, mediante la aplicación de entrevistas y encuestas a empleadores (Rehman y otros, 1998; Aina y Moahi, 1999; Mahmood, 2003), a graduados (Buttlar y Mont , 1996 en Estados Unidos; Midleton, 2003 en Australia; Moreiro y otros, 2008 en España), y a profesionales, profesores y estudiantes (Rehman, 2003; Marouf y Rehman, 2007). Por último, diversos autores han llevado a cabo análisis de los anuncios de trabajo para conocer las competencias requeridas por el mercado (Kennan y otros, 2006; Gerolimos y Konsta, 2008; Moreiro y otros, 2009; Artaza, 2011; Oliva Marañón, 2012).
En este estudio analizamos la valoración que hacen los empleadores del sector de la documentación, archivos y bibliotecas, sobre la importancia que tiene para ellos una serie de competencias referidas al máster en Gestión de la Documentación, Bibliotecas y Archivos de la Universidad Complutense de Madrid. También se analiza la opinión de los profesores y estudiantes participantes en el máster sobre esas mismas competencias; en el caso de los profesores, además, se les solicitó que indicaran el grado en el que, según ellos, éstas se encuentran incluidas en el plan de estudios $y$, en el caso de los estudiantes, se les solicitó que expresaran su grado de satisfacción con el aprendizaje de cada una de ellas.

Las competencias fueron seleccionadas a partir de dos fuentes documentales: en primer lugar, se utilizó el documento Verifica, aprobado por la ANECA, en el que aparecen competencias generales, transversales y específicas del título de máster (http://documentacion.ucm.es/master). Dado el elevado número en el que éstas aparecen en dicho documento, se eligieron aquéllas más acordes con los perfiles profesionales que se requieren actualmente en nuestro ámbito, es decir, las competencias referidas a la gestión de la información, a las aplicaciones de las tecnologías de la información y la comunicación, a los atributos personales y sociales que debe poseer un profesional, así como las necesarias para la gestión de unidades de información. En segundo lugar, tuvimos en cuenta también un conjunto de competencias de carácter más tecnológico relacionadas con la arquitectura de sistemas, aplicaciones web, redes de comunicación, o aplicaciones de la informática a la gestión documental, las cuales se seleccionaron a partir del listado de competencias que obtuvieron Marouf y Rheman (2007), utilizando un grupo focal en el que participaron empleadores, profesionales, profesores y estudiantes, con el objetivo de mejorar los contenidos de un Máster en LIS ofertado por la Universidad de Kuwait.

\section{METODOLOGÍA}

Para la realización del estudio se elaboró un cuestionario que incluía un conjunto de 29 competencias. En mayo de 2011 fue enviado a organizaciones del sector de la documentación (bibliotecas, archivos y centros de documentación) para que los profesionales valorasen la importancia de la presencia de dichas competencias en el Programa de Máster. El mismo cuestionario se utilizó para los profesores y los estudiantes; además, en el caso de los profesores, se les pidió que señalaran el grado en el que éstas aparecían en la disciplina que impartían y, en el caso de los estudiantes, que valorasen el grado en el que dichas competencias estaban incluidas en el programa. La escala de valoración utilizada fue del 1 al 10, siendo 10 el valor más alto.

La muestra se seleccionó a partir de diversas fuentes tomando como referencia aquellas organi- 
zaciones más cercanas con el público objetivo del máster. En el caso de los directores de empresas de servicios, la información se obtuvo en la página web http://www.docuweb.es/empresas/. Con respecto a los centros de documentación, se seleccionaron aquéllos pertenecientes a los diarios y cadenas de televisión de alcance nacional. En el caso de los archivos, los cuestionarios se enviaron a los responsables de los archivos generales institucionales y de comunidades autónomas. Por último, se seleccionó una muestra de bibliotecas provinciales y de universidades.

El total de organizaciones fue de 220. A cada una de ellas, se le envió por correo postal el cuestionario acompañado de una carta en la que se les explicaba el proyecto, así como un sobre de respuesta. Del total de organizaciones encuestadas obtuvimos respuesta de $68(30,9 \%)$, la mayor parte de las contestaciones procedieron de directores de bibliotecas y archivos $(58,4 \%)$.

Los estudiantes entrevistados fueron aquéllos que estaban matriculados en el máster durante el curso 2012-2013. De un total de 54, cumplimentaron correctamente el cuestionario 45 (83\%). En cuanto a los profesores, se recogió la opinión de 21 que impartían diferentes materias.

Los datos recogidos de los cuestionarios fueron procesados mediante la versión 19 del programa estadístico IBM-SPSS. De cada ítem se calculó el valor medio y la desviación estándar, y con el fin de averiguar si existían diferencias en las valoraciones que realizaron los diferentes grupos encuestados, se llevaron a cabo tres contrastes de hipótesis. El primer test de hipótesis tenía por objetivo contrastar la existencia o no de diferencias en las valoraciones de cada atributo presentado en el cuestionario, para ello, se consideró un nivel de confianza del $95 \%(a=0.05)$ para los valores medios de importancia. Dado que el número de encuestados en cada grupo (excepto en el de profesores) era superior a 30, se aplicó el Teorema Central del Límite para el cálculo del intervalo de confianza. El segundo test de hipótesis tenía por objetivo contrastar la existencia o no de diferencias en las valoraciones de cada atributo presentado en el cuestionario en el caso de los profesores del máster durante el curso 2012-2013. Dado que el número de encuestados era inferior a 30 , se utilizó la prueba de la T de Student.

El tercer test de hipótesis empleado, tenía como objetivo contrastar la existencia o no de diferencias entre los valores promedios globales de las competencias incluidas en cada factor. Los intervalos de confianza se construyeron siguiendo la metodología aplicada por Wu y Shieh (2009), para el caso de varianzas distintas.

Con el objeto de conocer si las competencias recogidas en el cuestionario se podían agrupar en diferentes categorías, se realizó un análisis factorial mediante el método de componentes principales con rotación varimax y normalización Kaiser. Tras diversas pruebas, se seleccionó una solución de cuatro factores que explican más del 59,5\% de la variabilidad total de la varianza, valor aceptable para las ciencias sociales (Hair y otros, 2010). Así mismo, el valor de 0,841 de la medida de adecuación muestral KMO, junto con el de los coeficientes alfa de Cronbach para el total de preguntas y para cada factor que fueron superiores a 0,80 , indican que los resultados que a continuación se analizan son adecuados y que, por tanto, las competencias se pueden agrupar en cuatro categorías.

\section{RESULTADOS}

Los resultados obtenidos para cada factor en cada una de las muestras analizadas son los siguientes:

\section{Importancia de las competencias en ges- tión de la información}

Las competencias que se incluyen en este factor aparecen relacionadas en la tabla I. Como se puede observar, reciben en general unas altas puntuaciones promedio por parte de los tres grupos de estudio (superiores a 7,85 sobre 10). Es de destacar la existencia de diferencias estadísticamente significativas (nivel de probabilidad del 95\%) entre las puntuaciones promedio que hacen empleadores y estudiantes con respecto a la competencia de "conocimientos en gestión de bases de datos", en el que los estudiantes tienen una opinión más favorable que los empleadores.

\section{Importancia de las competencias en co- municación e interrelación con compañeros y usuarios}

A la vista de la información que se presenta en la tabla II podemos señalar que la mayoría de las competencias que conforman esta categoría son altamente valoradas (valores medios superiores a $8,40$ sobre 10$)$. Es de destacar la alta valoración que otorgan los empleadores a la competencia de "capacidad para formar, coordinar y dirigir equi-

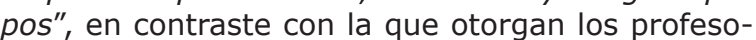
res y estudiantes, aunque dicha diferencia no sea estadísticamente significativa. Lo contrario sucede con la "capacidad de comunicación oral y escrita en inglés", que es peor valorada por los empleadores que por estudiantes y profesores

\section{Importancia de las competencias relacio- nadas con la informática y las aplicaciones de las tecnologías electrónicas}

De la observación de los datos que se presentan en la tabla III podemos concluir, en primer lugar, que las puntuaciones promedio de las competencias que se incluyen en este factor son, en general, más bajas que las que se obtienen en el resto de los factores, sobre todo, en el caso de los em- 
Tabla I. Valoración por los empleadores, estudiantes y profesores de la importancia de las competencias incluidas en el Factor 1 (valores medios y desviación estándar)

\begin{tabular}{|c|c|c|c|}
\hline Competencias incluidas en el Factor 1 & $\begin{array}{l}\text { Empleadores } \\
\text { Media(d.e.) }\end{array}$ & $\begin{array}{l}\text { Estudiantes } \\
\text { Media(d.e.) }\end{array}$ & $\begin{array}{l}\text { Profesores } \\
\text { Media(d.e.) }\end{array}$ \\
\hline Conocimiento sobre gestión de contenidos & $8,26(1,18)$ & $8,60(1,24)$ & $8,58(1,26)$ \\
\hline $\begin{array}{l}\text { Conocimiento de las fuentes de información, recuperación y } \\
\text { almacenamiento de la misma }\end{array}$ & $8,82(1,20)$ & $8,67(1,25)$ & $9,00(1,11)$ \\
\hline Capacidad para analizar y sintetizar información & $8,72(1,06)$ & $8,60(1,29)$ & $9,16(0,90)$ \\
\hline Conocimientos en gestión de bases de datos & $7,94(1,58)$ & $8,77(1,09)$ & $8,21(1,96)$ \\
\hline Capacidad para gestionar sistemas de gestión y control de la información & $8,12(1,10)$ & $8,14(1,26)$ & $8,11(1,37)$ \\
\hline Conocimiento de los proveedores y usuarios de la información & $7,85(1,64)$ & $8,28(1,53)$ & $8,00(1,49)$ \\
\hline Capacidad de planificación de sistemas de información & $8,16(1,32)$ & $7,98(1,34)$ & $8,16(1,12)$ \\
\hline $\begin{array}{l}\text { Conocimiento de los métodos normalizados de descripción, } \\
\text { presentación y transmisión de la información }\end{array}$ & $8,19(1,43)$ & $8,74(1,33)$ & $8,84(0,96)$ \\
\hline $\begin{array}{l}\text { Conocimiento para navegar por las redes de comunicación mediante } \\
\text { motores de búsqueda y otras herramientas }\end{array}$ & $8,32(1,43)$ & $8,44(1,56)$ & $8,95(0,85)$ \\
\hline
\end{tabular}

(d.e.) desviación estándar

Tabla II. Valoración por los empleadores, estudiantes y profesores de la importancia de las competencias incluidas en el Factor 2 (valores medios y desviación estándar)

\begin{tabular}{lccc}
\hline Competencias incluidas en el Factor $\mathbf{2}$ & $\begin{array}{c}\text { Empleadores } \\
\text { Media(d.e.) }\end{array}$ & $\begin{array}{c}\text { Estudiantes } \\
\text { Media(d.e.) }\end{array}$ & $\begin{array}{c}\text { Profesores } \\
\text { Media(d.e.) }\end{array}$ \\
\hline Habilidades de interrelación social con los compañeros y superiores & $8,60(1,63)$ & $8,72(1,30)$ & $8,37(1,38)$ \\
\hline Capacidad para trabajar en equipo & $9,00(1,32)$ & $8,91(1,31)$ & $8,74(1,05)$ \\
\hline Capacidad y habilidad para interrelacionar con los usuarios & $9,04(1,30)$ & $9,05(1,09)$ & $8,68(1,16)$ \\
\hline Capacidad de comunicación oral y escrita en el propio idioma & $8,72(1,34)$ & $8,95(1,33)$ & $8,84(1,17)$ \\
\hline Capacidad de transferencia del conocimiento & $8,47(1,26)$ & $8,26(1,31)$ & $8,21(1,40)$ \\
\hline Capacidad para formar, coordinar y dirigir equipos & $8,54(1,52)$ & $7,84(1,49)$ & $7,47(1,90)$ \\
\hline Capacidad de comunicación oral y escrita en inglés & $7,56(1,60)$ & $8,00(1,50)$ & $8,111,10)$ \\
\hline
\end{tabular}

(d.e.) desviación estándar

pleadores. En segundo lugar, que las puntuaciones promedio que se obtienen para cada competencia por parte de los empleadores es inferior a la de los estudiantes, aunque esta diferencia solo es significativa (nivel de probabilidad del 95\%) para:

"Dominio de aplicaciones web".

"Conocimientos técnicos suficientes para resolver problemas imprevistos".

"Conocimientos sobre arquitectura de sistemas".

\section{Importancia de las competencias relacio- nadas con la gestión de la organización}

De la observación de la tabla IV se infiere que las puntuaciones promedio sobre la importancia de las competencias que se incluyen en este factor presentan un amplio rango de variación, sobre todo en el caso de los empleadores (rango 6,81 a 9,07). Es de destacar que la "capacidad de resolución de problemas" es la competencia más valorada por empleadores y estudiantes. Así mismo, la competencia peor valorada por empleadores y profesores es la de "capacidad para gestionar e implementar políticas, códigos deontológicos, sociales y legales". En el caso de estudiantes la peor valorada es la "capacidad para gestionar recursos humanos y financieros".

\section{Diferencias en las valoraciones de impor- tancia de cada factor}

En la tabla $V$ se han dispuesto los valores medios agregados de las opiniones sobre la importancia de las competencias incluidas en cada uno de los factores, así como los intervalos de confianza con un nivel de probabilidad del 95\% para dichos valores. 
Tabla III. Valoración por los empleadores, estudiantes y profesores de la importancia de las competencias incluidas en el Factor 3 (valores medios y desviación estándar)

\begin{tabular}{|c|c|c|c|}
\hline Competencias incluidas en el Factor 3 & $\begin{array}{l}\text { Empleadores } \\
\text { Media(d.e.) }\end{array}$ & $\begin{array}{l}\text { Estudiantes } \\
\text { Media(d.e.) }\end{array}$ & $\begin{array}{l}\text { Profesores } \\
\text { Media(d.e.) }\end{array}$ \\
\hline Dominio de aplicaciones web & $7,54(1,42)$ & $8,44(1,30)$ & $8,37(1,16)$ \\
\hline $\begin{array}{l}\text { Conocimientos de recursos y aplicaciones electrónicas para gestionar } \\
\text { un proceso técnico cambiante }\end{array}$ & $7,66(1,55)$ & $8,42(1,45)$ & $7,74(1,97)$ \\
\hline $\begin{array}{l}\text { Conocimientos técnicos suficientes para resolver problemas } \\
\text { imprevistos }\end{array}$ & $7,51(1,67)$ & $8,51(1,40)$ & $8,16(1,38)$ \\
\hline Conocimientos sobre arquitectura de sistemas & $6,06(1,93)$ & $7,16(1,63)$ & $6,26(2,28)$ \\
\hline Conocimientos sobre gestión y diseño de Intranet y páginas web & $6,94(1,66)$ & $7,72(1,67)$ & $7,42(2,14)$ \\
\hline Capacidad para implementar un sistema de gestión & $7,85(1,64)$ & $8,12(1,42)$ & $7,74(1,94)$ \\
\hline
\end{tabular}

(d.e.) desviación estándar

Tabla IV. Valoración por los empleadores, estudiantes y profesores de la importancia de las competencias incluidas en el Factor 4 (valores medios y desviación estándar)

\begin{tabular}{lccc}
\hline Competencias incluidas en el Factor 4 & $\begin{array}{c}\text { Empleadores } \\
\text { Media(d.e.) }\end{array}$ & $\begin{array}{c}\text { Estudiantes } \\
\text { Media(d.e.) }\end{array}$ & $\begin{array}{c}\text { Profesores } \\
\text { Media(d.e.) }\end{array}$ \\
\hline Capacidad para gestionar recursos humanos y financieros & $7,79(1,97)$ & $7,49(1,59)$ & $7,05(1,90)$ \\
\hline $\begin{array}{l}\text { Capacidad para gestionar e implementar políticas, códigos } \\
\text { deontológicos, sociales y legales }\end{array}$ & $6,81(2,08)$ & $7,58(1,45)$ & $7,05(1,31)$ \\
\hline Capacidad para crear y organizar servicios para el usuario & $8,93(1,27)$ & $8,40(1,40)$ & $8,47(1,35)$ \\
\hline Capacidad analítica para combinar y organizar la información compleja & $8,43(1,37)$ & $8,30(1,32)$ & $8,74(0,93)$ \\
\hline Capacidad para gestionar proyectos & $8,32(1,45)$ & $7,86(1,41)$ & $8,42(1,30)$ \\
\hline Capacidad de resolución de problemas & $9,07(1,32)$ & $8,86(1,06)$ & $8,58(0,84)$ \\
\hline Capacidad para analizar y organizar datos estadísticos & $7,13(1,93)$ & $7,56(1,42)$ & $7,63(1,30)$ \\
\hline
\end{tabular}

(d.e.) desviación estándar

Tabla V. Valoración por los empleadores, estudiantes y profesores del grado de importancia del total de competencias incluidas en cada factor

\begin{tabular}{lcccccc}
\hline \multirow{2}{*}{\multicolumn{1}{c}{ FACTORES }} & \multicolumn{2}{c}{ Empleadores } & \multicolumn{2}{c}{ Estudiantes } & \multicolumn{2}{c}{ Profesores } \\
\cline { 2 - 7 } & Media & IC* & Media & IC* & Media & IC* \\
\hline $1^{\circ}$. Gestión de la información & 8,27 & $8,37-8,16$ & 8,47 & $8,60-8,34$ & 8,56 & $8,75-8,37$ \\
\hline $2^{\circ}$. Comunicación e interrelación & 8,56 & $8,69-8,43$ & 8,53 & $8,68-8,38$ & 8,35 & $8,58-8,10$ \\
\hline $3^{\circ}$. Aplicación de tecnologías & 7,26 & $7,67-7,33$ & 8,06 & $8,24-7,88$ & 7,61 & $7,96-7,27$ \\
\hline $4^{\circ}$. Gestión en general & 8,07 & $8,22-7,92$ & 8,01 & $8,16-7,85$ & 7,99 & $8,22-7,77$ \\
\hline Valoración media global & 8,14 & $8,06-8,20$ & 8,29 & $8,21-8,36$ & 8,17 & $8,05-8,29$ \\
\hline
\end{tabular}

( IC*) Intervalo de confianza para la media para un nivel de confianza del 95,0\% 
De la observación de la tabla $V$, se pueden inferir los siguientes hechos:

Los estudiantes dan una valoración media global del nivel de importancia de las competencias significativamente mayor (nivel de confianza del $95 \%)$ que los empleadores $(8,29$ frente a 8,14$)$.

La existencia de diferencias significativas entre empleadores y estudiantes (nivel de confianza del $95 \%$ ) en el valor medio agregado del factor 3 , que incluye las competencias relacionadas con la informática y las aplicaciones electrónicas.

El factor más importante para empleadores y estudiantes es el que incluye competencias relacionadas con la comunicación e interrelación con usuarios y compañeros (factor 2). En el caso de los empleadores su valor medio es superior a la valoración que hacen de los otros factores (nivel de confianza del 95\%). En el caso de los estudiantes la valoración media es superior a la de los factores 3 y 4 (nivel de probabilidad del 95\%).

Para los empleadores y profesores el factor menos importante es el de informática y aplicación de las tecnologías de la información y de la comunicación (factor 3). En el caso de los estudiantes es el factor 4 el que obtiene una valoración media más baja.

Para conocer si existen grandes diferencias en la opinión de empleadores, estudiantes y profesores, se han ordenado las competencias de acuerdo con su valor promedio. A partir de esta ordenación se han tomado como referencia las cinco competencias que son consideradas más importantes por los empleadores, y éstas se han comparado con el orden que presentan en las opiniones de estudiantes y profesores:

"Capacidad de resolución de problemas" (10 puesto en importancia para empleadores, 30 para estudiantes y $8^{\circ}$ para profesores).

"Capacidad y habilidad para interrelacionar con los usuarios" ( $2^{\circ}$ puesto en importancia para empleadores, 10 para estudiantes y 70 para profesores).
Capacidad para trabajar en equipo ( $3^{\circ}$ puesto en importancia para empleadores, $2^{\circ}$ para estudiantes y $4^{\circ}$ para profesores).

"Capacidad para crear y organizar servicios para los usuarios" (40 puesto en importancia para empleadores, $13^{\circ}$ para estudiantes y $11^{\circ}$ para profesores).

"Conocimiento de las fuentes de información, recuperación y almacenamiento" (50 puesto en importancia para empleadores, $8^{\circ}$ para estudiantes y $2^{\circ}$ para profesores).

En el caso de las competencias peor valoradas por los empleadores, los resultados son:

"Conocimientos sobre arquitectura de sistemas" (puesto 290 en importancia para empleadores, $29^{\circ}$ para estudiantes y $29^{\circ}$ para profesores).

"Capacidad para gestionar e implementar políticas, códigos deontológicos, sociales y legales" (puesto $28^{\circ}$ en importancia para empleadores, $26^{\circ}$ para estudiantes y $28^{\circ}$ para profesores).

"Conocimientos sobre gestión y diseño de intranet y páginas web" (puesto 270 en importancia para empleadores, $25^{\circ}$ para estudiantes y $25^{\circ}$ para profesores).

\section{Diferencias entre la valoración de las com- petencias que hacen los estudiantes, y la sa- tisfacción con la que se imparten en el Pro- grama Máster}

Como se observa en la figura 1 los estudiantes valoran peor el resultado del aprendizaje de las competencias que la importancia que tienen para ellos. Los valores promedios obtenidos para cada una de las competencias no son iguales con un nivel de confianza del $95 \%$, excepto en el caso de "Capacidad para gestionar proyectos" incluida en el factor 4.

Figura 1. Valores promedios de la opinión de los estudiantes sobre la importancia de cada competencia y el nivel de satisfacción que tienen con su aprendizaje en el máster

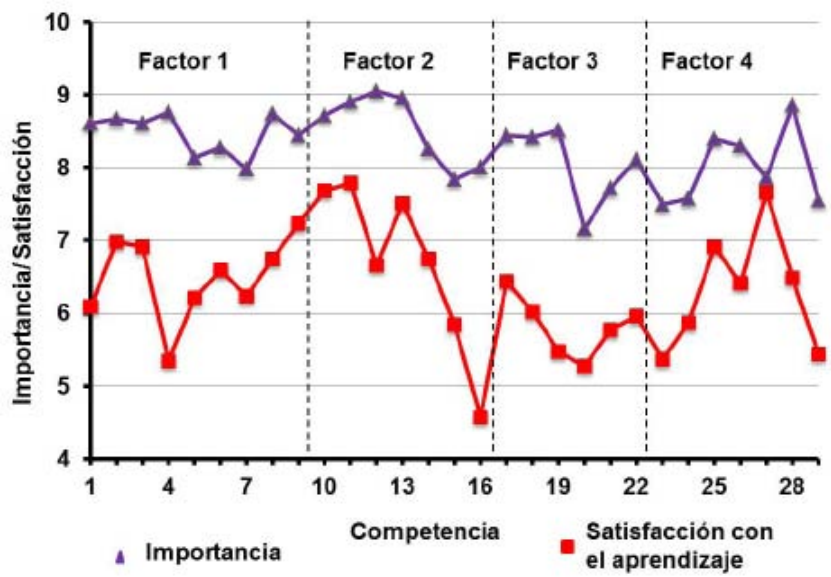


En la tabla VI se presentan las valoraciones promedio de importancia y satisfacción para cada uno de los factores. Como se puede observar, los estudiantes expresan su mayor satisfacción con el aprendizaje de las competencias incluidas en el factor 1 y 2 .

Además, es de destacar que las competencias incluidas en el factor 3 relacionado con la aplicación de las tecnologías de la información y la comunicación, son con las que los estudiantes opinan estar menos satisfechos en lo que se refiere a su aprendizaje.

Valores de satisfacción con el aprendizaje de las competencias superiores a 7, se obtiene para:

El conocimiento de las fuentes de información, recuperación y almacenamiento de la misma.

Capacidad para analizar y sintetizar información.

Habilidades de interrelación social con los compañeros y superiores.

Capacidad para trabajar en equipo.

Capacidad de comunicación oral y escrita en el propio idioma.

Capacidad para gestionar proyectos.
Conocimiento para navegar por las redes de comunicación mediante motores de búsqueda y otras herramientas.

Valores inferiores a 5,5 de promedio, en cuanto al grado en el que se adquiere una competencia, los encontramos en:

"Capacidad de comunicación oral y escrita en inglés" satisfacción de 4,57.

"Conocimientos sobre arquitectura de sistemas", satisfacción de 5,27.

"Conocimientos en gestión de bases de datos", satisfacción de 5,34.

"Capacidad para gestionar recursos humanos y financieros", satisfacción de 5,36.

"Capacidad para analizar y organizar datos estadísticos", satisfacción de 5,43.

7. Diferencias entre la valoración de la importancia de las competencias que hacen los profesores, y su opinión sobre el grado de inclusión en el programa de máster

En la figura 2 se representan las puntuaciones de importancia otorgadas a cada una de las competencias por parte de los profesores, y la valoración que realizan sobre el grado en que dichas competencias se incluyen en el máster.

Tabla VI. Valores promedio agregados de la valoración de la importancia y la satisfacción con el aprendizaje de las competencias incluidas en cada factor según la opinión de los estudiantes

\begin{tabular}{lcccc}
\hline \multirow{2}{*}{ FACTORES } & \multicolumn{2}{c}{ Importancia } & \multicolumn{2}{c}{ Satisfacción } \\
\cline { 2 - 5 } & Media & IC* & Media & IC \\
\hline $1^{\circ}$. Gestión de la información & 8,47 & $8,60-8,34$ & 6,82 & $7,09-6,56$ \\
\hline $2^{\circ}$. Comunicación e interrelación & 8,53 & $8,68-8,38$ & 6,68 & $6,93-6,44$ \\
\hline $3^{\circ}$. Aplicación de tecnologías & 8,06 & $8,24-7,88$ & 5,82 & $6,12-5,53$ \\
\hline $4^{\circ}$. Gestión en general & 8,01 & $8,16-7,85$ & 6,30 & $6,53-6,07$ \\
\hline
\end{tabular}

( IC*) Intervalo de confianza para la media para un nivel de confianza del 95,0\%

Figura 2. Valores promedios de la opinión de empleadores sobre la importancia de cada competencia y el nivel de inclusión de la misma en el máster

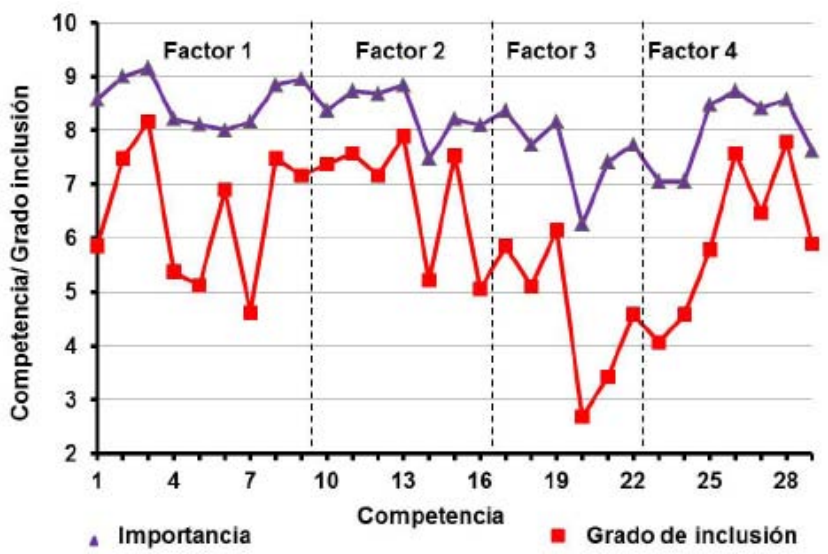


Como se puede observar, existen 11 competencias que, según la opinión de los profesores, tienen un gran peso en el máster (valores promedio superiores a 7); sin embargo, las competencias incluidas en el factor 3 , y que están relacionadas con la informática y las aplicaciones de las tecnologías de la información son, en opinión de los profesores, escasamente incluidas en el aprendizaje de los estudiantes. Dentro de este factor hay que destacar las bajas valoraciones de:

"Conocimientos sobre arquitectura de sistemas", que recibe una valoración promedio de 2,68.

"Conocimientos sobre gestión y diseño de intranet y páginas web", que recibe una valoración de 3,4 .

"Capacidad para implementar un sistema de gestión", que recibe una valoración de 4,58.

Valoraciones inferiores a 5 las encontramos también en las siguientes competencias:

"Capacidad para gestionar recursos humanos y financieros" $(4,05)$.

"Capacidad para gestionar e implementar políticas, códigos deontológicos, sociales y legales" $(4,57)$.

"Conocimiento de los métodos normalizados de descripción, presentación y transmisión de la información" $(4,61)$.

\section{CONCLUSIONES}

De acuerdo con los resultados de satisfacción de los estudiantes con el proceso de aprendizaje se constata, en primer lugar, que el Máster en Gestión de la Documentación, Bibliotecas y Archivos de la UCM incluye, de forma razonable, todas aquellas competencias relacionadas con la comunicación y las relaciones sociales, con el trabajo en grupo, la resolución de problemas y el enfoque a los usuarios, así como las incluidas dentro del epígrafe de gestión de la información. Además, las anteriores competencias son las que reciben una mayor valoración en su importancia por parte de empleadores, profesores y estudiantes. Así mismo, se constata que las competencias de carácter más informático como arquitectura de bases de datos, o conocimientos sobre gestión y diseño de Intranet y páginas web, son las que recibieron la más baja valoración.

En segundo lugar, hay que destacar que los estudiantes dieron una valoración media de satisfacción superior a 6 en 20 de las 29 competencias que se les presentaron, lo que sugiere que el programa ofertado es ligeramente satisfactorio para éstos a nivel global, a pesar de que, como se ha señalado antes, existe una amplia distancia entre la opinión expresada acerca de la importancia de las competencias y la satisfacción con el aprendizaje de éstas en el máster.
No obstante, el programa de máster podría mejorar notablemente si se implementaran acciones para incrementar la satisfacción en las siguientes competencias:

"Capacidad de comunicación oral y escrita en inglés", que presenta una satisfacción media con su aprendizaje por parte de los estudiantes de 4,57 , frente a una importancia de 8,00 , y en la que los profesores señalan, además, que no está bien representada en el programa. Tengamos en cuenta que esta capacidad es uno de los objetivos que tienen que conseguir los titulados dentro del EEES.

"Capacidad de resolución de problemas", que aunque recibe un valor de satisfacción favorable de 6,45 por parte de los estudiantes, sin embargo, las altas valoraciones que dan los empleadores $(9,06)$ y estudiantes $(8,89)$, debería llevar a la consideración de su mejora.

"Capacidad y habilidades para interrelacionar con los usuarios". El aprendizaje de esta competencia, por parte de los estudiantes, recibe una valoración de satisfacción de 6,65 frente a una valoración de importancia superior a 9,00 por parte de empleadores y estudiantes.

Con carácter general, podemos indicar que para mejorar los resultados en el aprendizaje de alguna de las anteriores competencias, sería necesario que el programa de máster tuviera una duración de, al menos, tres semestres, lo que permitiría implementar un "practicum" y la mejora en la adquisición de competencias de interrelación social y de comunicación, o de resolución de problemas dentro de un entorno de trabajo real.

A pesar de que el trabajo aquí presentado tiene limitaciones importantes en cuanto al número de empleadores encuestados, sin embargo, consideramos que esta es una aproximación útil para hacer el seguimiento de los resultados del aprendizaje de un Programa de Máster en Gestión de la Documentación, Bibliotecas y Archivos.

\section{BIBLIOGRAFÍA}

Aina, L. O.; Moahi, K. (1999).Employers' perceptions of the graduates and curriculum of a library school in Botswana. Libri, vol. 49, 175-179.

American Library Association. (2008). Standards for accreditation of master's programs in library and information studies. Disponible en: http:// www.ala.org/accreditedprograms/sites/ala.org. accreditedprograms/files/content/standards/ standards_2008.pdf: (Consultado 5 de junio de 2013).

Artaza, C. H. (2011).El mercado laboral de los titulados en Bibliotecología y Documentación: análisis de las ofertas de empleo publicadas en Argentina. Investigación Bibliotecológica, vol. 25 (54), 17-36. 
Buttlar, L.; Du Mont, R. (1996). Library and information science competencies revisited, Journal of Education for Library and Information Science, vol. 37, 44-62. http://dx.doi. org/10.2307/40324283 (Consultado 5 de junio de 2013)

Gerolimos, M.; Konsta, R. (2008). Librarians' skills and qualifications in a modern informational environment. Library Management, vol. 29, (8/9), 691-699. http://dx.doi. org/10.1108/01435120810917305 (Consultado 5 de junio de 2013)

Hair, J. F.; Black, W. C.; Babin, B. J.; Anderson, R. E. (2010).Multivariate Data Analysis. Seventh Edition. Prentice Hall.

IFLA (International Federation of Library Associations and Institutions). Education and Training Section. Guidelines for professional library/information educational programs - 2000.Third revised draft, 2003. Disponible en: http://www. ifla.org/VII/s23/bulletin/guidelines.htm (Consultado 17 de junio de 2013).

Kennan, M. A; Willard, P; Wilson, C.S. (2006). What do they want? - a study of changing employer expectations of information professionals. Academic and Research Libraries. vol. 37 (1), 17-37. http://dx.doi.org/10.1080/00048623.20 06.10755320 (Consultado 17 de junio de 2013).

Mahmood, K. (2003). A comparison between needed competencies of academic librarians and LIS curricula in Pakistan. The Electronic Library. vol. 21 (2), 99-109. http://dx.doi. org/10.1108/02640470310462434 (Consultado 17 de junio de 2013).

Marouf, L.; Ur Rehman, S. (2007). New directions for information education: Perspectives of the stakeholders. Education for Information. vol. 25, 195-209.

Middleton, M. (2003).Skills expectations of library graduates. New Library World, vol.104 (1-2), 42-56. http://dx.doi. org/10.1108/03074800310458287 (Consultado 17 de junio de 2013).

Moreiro, J. A; Azcárate, P.; Marzal, M., Tejada, C. y Vergueiro, W. (2008). Desarrollo profesional y opinión sobre la formación recibida de los titulados universitarios en información y documentación de las universidades públicas de Madrid (2000-2005). El Profesional de la Información, 17 (3) 261-272. http://dx.doi. org/10.3145/epi.2008.may.02 (Consultado 17 de junio de 2013).

Moreiro, J. A.; Sanchez-Cuadrado, S.; Morato, J.; Moreno, V. (2009). Desarrollo de una aplicación ontológica para evaluar el mercado de trabajo español en Biblioteconomía y documentación. Revista Española de Documentación Científica, vol. 32 (1), 81-98. http://redc.revistas.csic.es/ index.php/redc/article/view/454/466 (Consultado 17 de junio de 2013)

Oliva Marañón, C. (2012). Competencias y formación universitaria del documentalista en el Espacio Europeo de Educación Superior (EEES): estudio de su adecuación al entorno laboral. Investigación Bibliotecológica, vol. 26 (54), 15-45.

Rehman, S. (2003). Information studies curriculum based on competency definition. Journal of Education for Library and Information Science, vol. 44, 276-295.

Rehman, S.; Baker, A. B. A.; Majid, S. (1998). Needed capabilities and development strategies for future information professionals: A Malaysian perspective. International Information \& Library Review, vol. 30, 123-141. http://dx.doi. org/10.1006/iilr.1998.0088 (Consultado 17 de junio de 2013).

Rehman, S.; Al-Ansari, H.; Yousef, N. (2002). Coverage of competencies in the curriculum of information studies: An international perspective. Education for information, vol. 20, 199-215.

SEDIC. (2005). Euro-referencial en información y documentación. Madrid: SEDIC.Disponible en: http://www.certidoc.net/es1/euref1-espanol. pdf (Consultado: 2 de abril de 2013).

Special Libraries Association. (2003). Competencies for Information Professionals of the 21 Century (revised edition June 2003). Disponible en: http://www.sla.org/about-sla/competencies/ (consultado 10 de junio de 2013).

Tejada, C.; Meyriat, J. (2003). Competencias en información y documentación. Los proyectos europeos DECIDOC y CERTIDOC. Educación y Biblioteca, vol. 137 (septiembre/octubre), 110-116.

Wu, H. H.; Shie, J. I. (2009). The development of a confidence interval- based importance-performance analysis by considering variability in analysing service quality. Expert Systems Applications, vol. 36 (4), 7040-7044. http://dx.doi. org/10.1016/j.eswa.2008.08.055 (Consultado 10 de junio de 2013). 